\title{
Sterile neutrino searches with the ICARUS detector
}

\author{
Gian Luca Raselli* \\ on behalf of the ICARUS Collaboration \\ INFN Sezione di Pavia \\ E-mail: gianluca.raselli@pv.infn.it
}

The Short Baseline Neutrino (SBN) Program is a short-baseline neutrino oscillation experiment to definitely prove or exclude the existence of non-standard neutrino oscillations into sterile states at the $1 \mathrm{eV}$ mass range. It consists of three Liquid Argon Time Projection Chamber (LArTPC) detectors deployed along the Booster Neutrino Beam-line (BNB) at Fermilab. The LArTPC technology has been taken to full maturity with ICARUS T600. After the completion of the overhauling at CERN, the apparatus was trasferred to FNAL where it will act as far detector. The program foresees the data taking for three years.

Neutrino Oscillation Workshop (NOW2018)

9 - 16 September, 2018

Rosa Marina (Ostuni, Brindisi, Italy)

${ }^{*}$ Speaker. 


\section{The Short Baseline Neutrino (SBN) program at FNAL}

Experimental observations of neutrino oscillations have so far established a standard paradigm accounting for three neutrino flavors $\left(v_{e}, v_{\mu}, v_{\tau}\right)$ resulting from the mixing of three mass eigenstates $\left(v_{1}, v_{2}, v_{3}\right)$. Nevertheless, some experiments have reported short-baseline $v$ oscillation anomalies [1] pointing toward the possible existence of non standard heavier sterile neutrino state(s) driving oscillations at small distances with $\Delta m^{2} \approx 1 \mathrm{eV}^{2}$ and relatively small mixing angles.

Since each of these measurements lacks the significance to claim a discovery, a Short Baseline Neutrino (SBN) program is being developed at Fermi National Accelerator Laboratory (FNAL) [2] to give a definitive answer to the "sterile neutrino puzzle", It foresees the deployment along the Booster Neutrino Beam (BNB) of three detectors, all based on the Liquid Argon Time Projection Chamber (LAr-TPC) technique. SBND, with $112 \mathrm{t}$ active volume, will be placed at $110 \mathrm{~m}$ from the target and will act as the "near detector"; MicroBooNE, with $89 \mathrm{t}$ active volume, taking data at $470 \mathrm{~m}$ from the neutrino source, will run as "intermediate detector"; ICARUS T600, with $476 \mathrm{t}$ active volume and set at a distance of $600 \mathrm{~m}$, will operate as "far detector". Such configuration will allow simultaneous observations of neutrino interactions at different distances, by independently measuring both $v_{e}$ appearance and $v_{\mu}$ disappearance oscillation channels. Any difference in the neutrino spectra at the different detectors might imply a new physics signal. Sensitivity studies and MC simulations demonstrate the possibility to cover the anomaly parameter region with a $5 \sigma$ confidence level in three years of data taking $\left(\approx 6.6 \times 10^{20}\right.$ pot $)$ by all three LAr-TPC detectors.

\section{ICARUS T600 and its performance at LNGS}

LAr-TPC detectors are well suited for experimental studies of neutrino physics, combining a massive and homogeneous target with excellent tracking and calorimetric capabilities. Originally proposed by C. Rubbia in 1977 [CERN-EP/77-08], this technology has been taken to full maturity with ICARUS T600, which still represents the largest LAr-TPC ever operated in a neutrino beam.

The T600 detector is made of two identical cryostats each housing two faced TPCs sharing a common cathode [3]. They are filled with a total of $760 \mathrm{t}$ of ultrapure liquid argon corresponding to $470 \mathrm{t}$ of active mass. Each TPC has a maximal drift length of $1.5 \mathrm{~m}$, over which a constant electric field $E=500 \mathrm{~V} / \mathrm{cm}$ is present.

Charged particles interacting in the TPC produce both scintillation light and ionization electrons: the latter are drifted by the field to the anode, which is made by three parallel wire planes with different orientation $\left(0^{\circ} ; \pm 60^{\circ}\right), 3 \mathrm{~mm}$ apart and with a $3 \mathrm{~mm}$ pitch. The LAr scintillation light is detected by mens of 8 in photomultiplier tubes (PMTs), able to operate at cryogenic temperature and made sensitive to VUV photons $(\lambda=128 \mathrm{~nm})$ by means of a wavelengh shifter (TPB) deposited on the PMT windows.

The measured drift time, combined with the electron drift velocity $(v \approx 1.6 \mathrm{~mm} / \mu \mathrm{s}$ at $E=$ $500 \mathrm{~V} / \mathrm{cm}$ ) provides the event coordinate in the drift direction; the composition of the three views from the TPC wires yields the track projection on the anode plane. This information allows obtaining a full 3D reconstruction of the tracks, with a spatial resolution of $\approx 1 \mathrm{~mm}^{3}$.

ICARUS T600 took data from 2010 to 2013 in the Gran Sasso Laboratory (LNGS, Italy), both with atmospheric neutrinos and with CNGS beam from CERN. The search for possible long- 
baseline sterile neutrino oscillations resulted in no evidence of $v_{e}$ excess above the expected beam contaminations [4].

During the LNGS run, important LAr-TPC features were demonstrated: the capability to attain unprecedented levels of liquid argon purity [5], the achievement of high efficient electron/gamma separation and the remarkable particle identification capability by exploiting the measurement of $d E / d x$ versus range [4], the possibility to measure the muon momentum by multiple scattering [6].

\section{ICARUS T600 overhauling at CERN}

From 2015 to 2017 the T600 detector underwent a significant overhauling process introducing new technological developments while at the same time maintaining the already achieved performance. This process took place at CERN in the framework of the Neutrino Platform activities (WA104/NP01). The overhauling covered important technical sections of the T600, such as: 1) the preparation of new cold vessels and of a new purely passive insulation system; 2) the refurbishing of the inner detectors with TPC cathodes with better planarity; 3) the installation of an improved scintillation light detection system; 4) a new, fast, higher-performance read-out electronics.

The scintillation light detection system was significantly upgraded by adopting 360 highperformance Hamamatsu R5912-MOD PMTs (8 in diameter) [7]. A total of 360 devices were mounted behind the 4 wire planes (90 each) allowing high detection coverage and sensitivity (below $100 \mathrm{MeV}$ ) and a time resolution better than $1 \mathrm{~ns}$, thanks to a laser calibration system.

A new compact read-out electronics was designed to allow continuous readout, digitization and independent waveform recording of signals from each wire of the TPC. It is based on the adoption of serial 12-bit ADCs with synchronous sampling (400 ns) of all the channels of the whole detector [8]
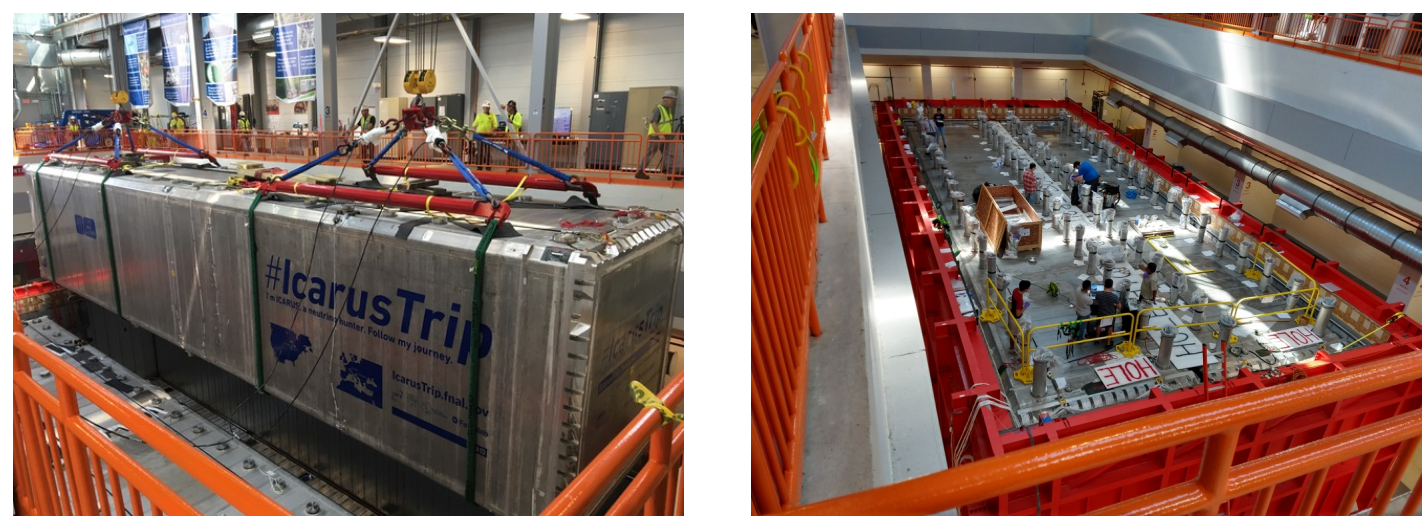

Figure 1: Pictures showing the final rigging of the ICARUS detector inside the far building (left) and the beginning of the assembly phase (right).

\section{Transportation to FNAL and present status}

In summer 2017 the detector was transferred to the U.S.A. with a special transport that took six weeks across the ocean, rivers and on the road. In the meantime, the civil construction of the far 
site and building was completed as well the assembly of the warm vessel floor/walls and the cold shields.

After the arrival at FNAL, the two modules were prepared for the final movement in the pit inside the far building. The rigging, shown in figure 1 left, took place in July 2018. From August 2018 the set up of the detector instrumentation is in progress with the assembly of all the external parts of the apparatus (figure 1 right). This phase will be followed in early 2019 by the commissioning and data taking which will last at least for three years.

\section{Conclusions}

Fifty years after their introduction by B. Pontecorvo, sterile neutrios are still an open question in particle physics. The SBN program, in preparation at FNAL and consisting of the SBND, MicroBooNE, and ICARUS LAr-TPC detectors, is expected to definitely prove or exclude their existence and to find a solution to the observed short-baseline anomalies. The LArTPC detector technology, necessary for the SBN experiment, has been taken to full maturity with ICARUS T600 which will be operated as far detector at $600 \mathrm{~m}$ from the neutrino source. The present anomaly parameter region will be covered with a $5 \sigma$ confidence level in three years of data taking with the three LArTPCs.

\section{References}

[1] I. Martinez-Soler, Global status of three-neutrino mixing, this conference; Z. Wang Reactor neutrino oscillations, this conference; M. Shaevitz Updated electron $n$ appearance results from MiniBooNE, this conference.

[2] R Acciarri et al., A Proposal for a Three Detector Short-Baseline Neutrino Oscillation Program in the Fermilab Booster Neutrino Beam, FNAL Proposal P-1052 (2014); arXiv:1503.01520.

[3] S. Amerio et al., Design, construction and tests of the ICARUS T600 detector, Nucl. Instrum. Meth. A 527 (2004) 329.

[4] M. Antonello et al., Experimental search for the "LSND anomaly" with the ICARUS detector in the CNGS neutrino beam, Eur. Phys. J. C73 (2013) 2345.

[5] M. Antonello et al., Experimental observation of an extremely high electron lifetime with the ICARUS-T600 LAr-TPC, JINST 9 (2014) P12006.

[6] M. Antonello et al., Muon momentum measurement in ICARUS-T600 LAr-TPC via multiple scattering in few-GeV range, JINST 12 (2017) P04010.

[7] M. Babicz et al., Test and characterization of 400 Hamamatsu R5912-MOD photomultiplier tubes for the ICARUS T600 detector, JINST 13 (2018) P10030.

[8] L. Bagby et al., New read-out electronics for ICARUS-T600 liquid Argon TPC. Description, simulation and tests of the new front-end and ADC system, JINST 13 (2018) P12007. 